\title{
Analysis of Heart Rate variability (HRV) in Frequency Domain and Recurrence Quantification Analysis during the Treatment by Transcutaneous Vagus Nerve Stimulation
}

\author{
Conte $\mathrm{S}^{1}$, Wang $\mathrm{F}^{2}$, Altamura $\mathrm{M}^{1,3}$, Bellomo \\ $A^{3}$, Serafini $G^{4}$, Orsucci $F^{5}$, Kaleagasioglu $F^{1,6}$, \\ Mendolicchio $L^{1,7}$, Casciaro $F^{1,8}$, Norman $R^{1}$ and \\ Conte $\mathrm{E}^{1 *}$ \\ ${ }^{1}$ School of Advanced International Studies on Applied \\ Theoretical and non Linear Methodologies of Physics, \\ Italy \\ ${ }^{2}$ College of Science/ Agricultural Mathematical Modeling \\ and Data Processing Center, Hunan Agricultural \\ University, China \\ ${ }^{3}$ Department of Biomedical Science, University of Foggia, \\ Italy \\ ${ }^{4}$ Tor Vergata University, Italy \\ ${ }^{5}$ Division of Psychology, University College, England \\ ${ }^{6}$ Faculty of Medicine, Near East University, Turkish \\ Republic of Northern Cyprus \\ ${ }^{7}$ Clinic Villa Miralago, Italy \\ ${ }^{8}$ Department of Biomedical Sciences and Human \\ Oncology, University of Bari, Italy \\ *Corresponding author: Conte E, School of Advanced \\ International Studies on Applied Theoretical and non \\ Linear Methodologies of Physics, Italy
}

Received: May 21, 2018; Accepted: June 18, 2018; Published: J une 25, 2018

\begin{abstract}
The aim of the present work has a methodological profile. It aims to analyze the HRV during thirty minutes transcutaneous Vagus Nerve Stimulation tVNS treatment. We used the linear methodology of the Fast Fourier Transform and an analysis of the variability of the R-R signal in frequency domain under such conditions of stimulation. As specialized method of analysis we used also the Recurrence Quantification Analysis (RQA). The importance to use a combination of such linear and non linear methodologies was to give results of physiological interest that is to say to ascertain, as well as possible, the influence of such tVNS treatment on HRV. We examined 50 young subjects in normal health conditions and 50 young subjects with Autonomic Nervous Dysfunction (ANS) as well as it is evidenced by HRV. The experiments were arranged in three phases, recording the data before, during, and after a 30-minute treatment. The analysis of the data was performed in the frequency domain evaluating the VLF, the LF, and the HF and the variability of the tachogram according to the 1996 Task Force Standards of the European Society of Cardiology and the North American Society of Pacing and Electrophysiology. In addition the Recurrence Quantification Analysis (RQA) was used. The indication arising from such methodological study is that the unbalanced initial ANS dysfunction of the examined subjects, as mirrored from the HRV analysis, compensates and balances with also a prevailing tendency to parasympathetic HRV modulation during the tVNS treatment.
\end{abstract}

Keywords: HRV; Transcutaneous Vagus Nerve Stimulation (tVNS); Ear clip and finger plethysmography; Fast Fourier Transform (FFT); Heart Rate Analysis of Variability; Heart Rate Analysis; Recurrence Quantification Analysis (RQA)

\section{Introduction}

The idea that we can influence neuronal activity with electrical pulses is not new. Earlier this century, patients were treated, and still are, with electroconvulsive therapy as a treatment for severe depression [1].

Presently, electrical stimulation of the Vagus Nerve (VNS) is an FDA-approved therapy tool for both refractory depression and epilepsy [2-4]. It has been used, as well, for a number of psychological and psychiatric disorders. Furthermore, it recently has emerged as a promising therapeutic approach for cardiac diseases $[5,6]$. As supported by neuroimaging studies, we also maintain that it broadly affects different parts of the brain, including the thalamus, cerebellum, orbito-frontal cortex, limbic system, hypothalamus, and medulla [7$10]$.

Traditionally, vagus nerve stimulation (VNS) has been performed by implanting a neurostimulating device connected to an electrode located along the cervical branch of the vagus nerve. In principle, this procedure may have negative aspects, such as coughing during stimulation, hoarseness, general operational and anesthesiological risks, and high costs. In order to minimize adverse effects, a new non-invasive neuro stimulating procedure, called transcutaneous Vagus Nerve Stimulation (tVNS) has been developed. We used the NuCalm device (http://www.nucalm.com/) which applies two gel electrode patches to the neck below the ear lobe (right and left) for transcutaneous stimulation of the afferent auricular branch of the vagus nerve $(\mathrm{ABVN})$. This device has received FDA approval, satisfying essential health and safety requirements. The employed tVNS targets the cutaneous receptive field of the ABVN at the outer ear [11]. Ear lobe vagal stimulation does not create adverse effects [12], but it does induce modulating effects on heart rate, blood pressure, or peripheral microcirculation during the stimulation procedure. The use of applied electrodes near both right and left ear lobes assures afferent and efferent induction since the right vagus nerve has efferent fibers to the heart. Of course, use of the device is not recommended in the case of pregnancy, and is contraindicated for persons with metal in the body, such as pacemaker and ventricular assist devices or brain and cochlear implants.

The aim of the present paper has a physiological-methodological profile, to evaluate the influence of the tVNS on the HRV during a
Ann Depress Anxiety - Volume 5 Issue 1 - 2018

ISSN : 2381-8883 | www.austinpublishing group.com

Conte et al. (C) All rights are reserved
Citation: Conte S, Wang F, Altamura M, Bellomo A, Serafini G, Orsucci F, et al. Analysis of Heart Rate variability (HRV) in Frequency Domain and Recurrence Quantification Analysis during the Treatment by Transcutaneous Vagus Nerve Stimulation. Ann Depress Anxiety. 2018; 5(1): 1093 


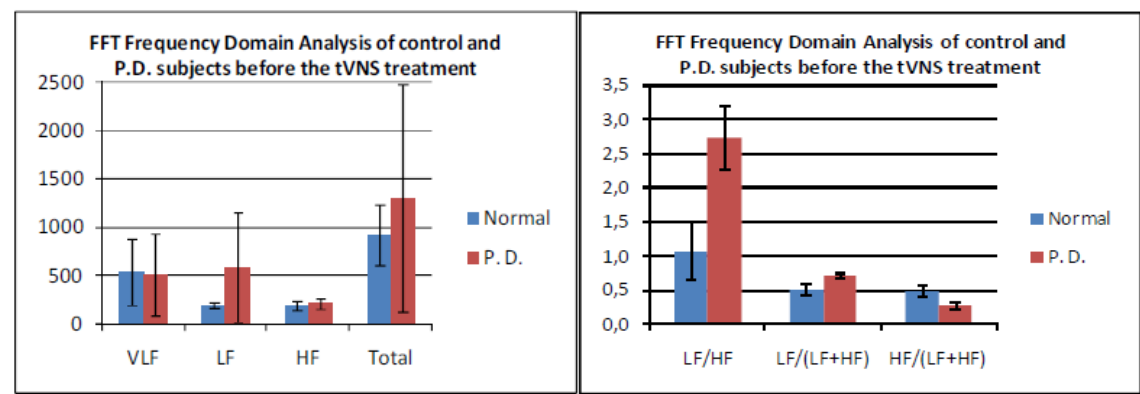

Figure 1: Compare normal subjects against subjects with psychological disorders (P.D.) using the standard FFT and CZF methods.
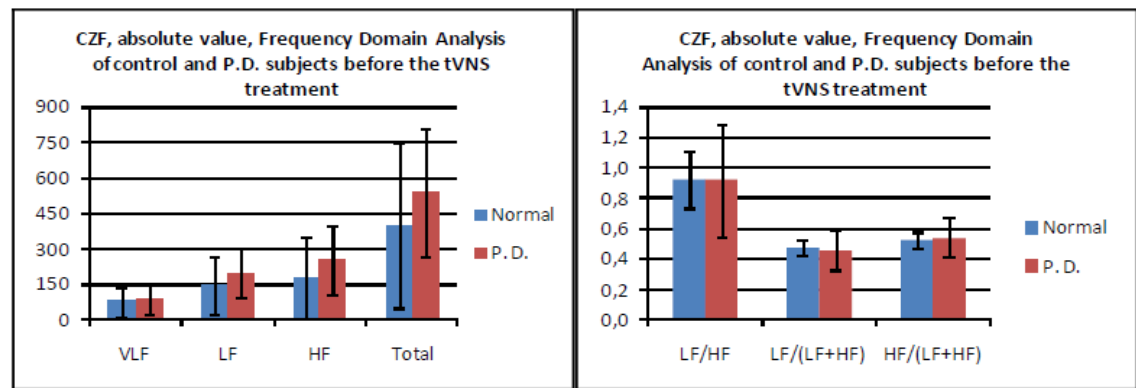

Figure 2: Comparison of normal subjects against subjects with psychological disorders (P.D.) using the standard FFT and CZF methods.

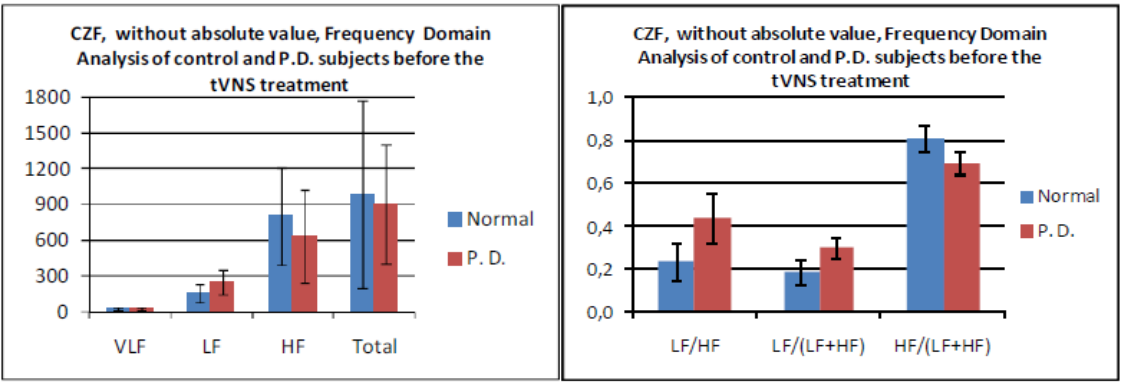

Figure 3: Examine the results without absolute value.

single treatment. As known, HRV is the modulation of heart rate dynamics through the Autonomic Nervous System (ANS) and is profoundly modified in a great variety of experimental conditions and, in particular, in subjects having Psychological Disorders (P.D.). A way to examine ANS dysfunction in P.D. is the frequency domain analysis of Fast Fourier Transform (FFT), which enables us to identify three bands in the obtained power spectrum frequency domain. These bands are the Very Low Frequency (VLF), the Low Frequency (LF), and the High Frequency, respectively. The LF and HF bands relate mainly to ortho-sympathetic and parasympathetic ANS modulation. Therefore, the focus of the present study was to select a group of P.D. subjects and investigate their HRV before, during and after the tVNS treatment, with the only and important goal of ascertaining whether this tNVS treatment really induces a modification of HRV in such subjects during such single treatment. We used different methods of linear analysis, in particular the R-R signal and the signal obtained from the R-R with estimation of the variability of such signal $[13,14]$ as well as clinicians need accurate determination in such frequency field when the tVNS treatment is acting. As general non linear method of analysis was added; it is the Recurrence Quantification Analysis (RQA). Such three employed methods of analysis were able to give a satisfactory evaluation of the acting ANS modulating dynamics accounting for linear and non linear physiological components.

\section{Materials and Methods}

The NuCalm device was furnished by Solace Life sciences, Inc., of Wilmington, Delaware, USA. Two groups of subjects were examined: 50 young subjects in normal health conditions and 50 young subjects affected by anxiety, or depression.

To test the subjects, each potential participant was given a nonstructured interview following the criteria of the Diagnostic and Statistical Manual of Mental Disorders (DSM). Participants selected for the study showed symptomatology of anxiety or depression. Those selected were divided into two groups and administered tests to evaluate the level of their anxiety and depression symptoms. The scales used to measure their scores were the Hamilton Anxiety Scale (HAM-A) and the Hamilton Depression Scale (HAM-D), 


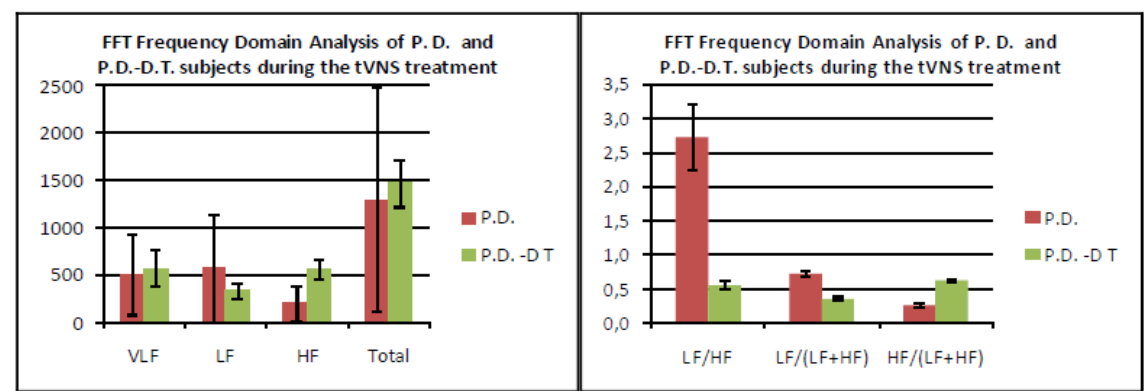

Figure 4: P.D.-DT represents results for P.D subjects during treatment.

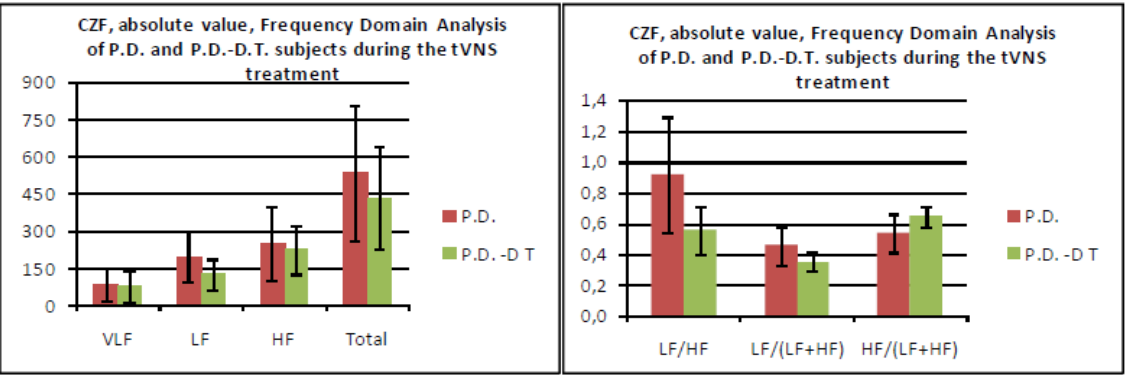

Figure 5: Let us examine now the results obtained by the CZF method.
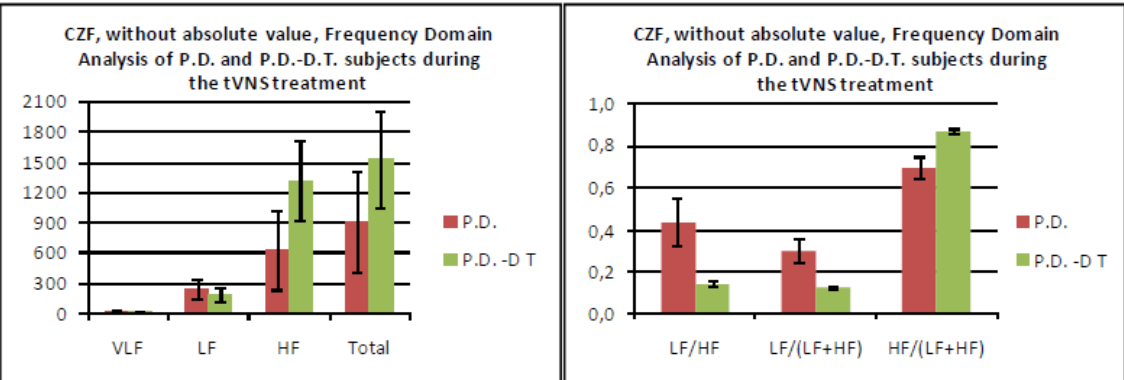

Figure 6: The results of the application to this case of the CZF method without absolute value thus accounting for acceleration and deceleration of the heart rhythm.

respectively. Both tests were administered during patient recruitment and showed medium scores, with HAM-A at 42 and HAM-D at 18 . Specifically, we examined subjects all having ages between 25 and 45 . All the subjects gave their written informed consent. According to the 1996 Task Force Standards of the European Society of Cardiology and the North American Society of Pacing and Electrophysiology, we selected the subjects excluding a priori those affected from well established other pathologies as we fixed in detail in [13].

The obtained tachograms were recorded by finger plethysmography (PG) and each time it was compared to those obtained by ECG, accounting for Pulse Wave Transit Time (PWTT).

The NuCalm device was employed for the transcutaneous stimulation, applying gel electrode patches near the right and left ear lobes, and setting the current value at 0.1 MA. The NuCalm device output was constantly monitored by a Welleman PCLAB 2000 SE computer interface. The scheme of the experiment was as follows. Each subject was recorded for three minutes by GSR in tone and phase values, just before the start of recording tachogram data. This was performed to establish the general condition of each subject. Soon after the experiment started a PG recording of five minutes was performed without tNVS. Then the PG recording was extended for 30 minutes with the subject under NuCalm stimulation. After 30 minutes of treatment, a final recording of five minutes was done on the subject without treatment. This scheme enabled us to estimate HRV before, during and after the tVNS.

\section{For analysis of the data, we used the following methods}

a) The fast Fourier Transform (FFT) spectrum analysis enabled us to estimate the VLF, LF and HF bands and the total power as frequency domain expression of the ANS modulation. This is the standard method, applied by re-sampling the original NN interval series and then applying the FFT. The VLF band was encoded between 0.003 and $0.04 \mathrm{~Hz}$, the LF band between 0.04 and $0.15 \mathrm{~Hz}$, and the HF band between 0.15 and $0.4 \mathrm{~Hz}$. It is known that the VLF spectral component $(0.003-0.05 \mathrm{~Hz})$ is possibly related to long-term regulatory mechanisms (e.g. the renin-angiotensin system, the thermoregulatory peripheral blood flow adjustment). The LF spectral component is 


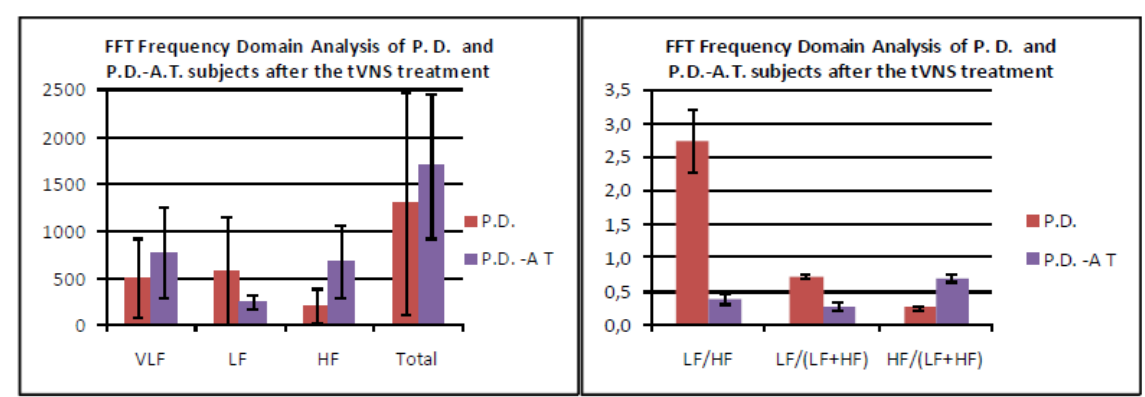

Figure 7: The results obtained after the tVNS treatment. Let us start with the standard FFT.

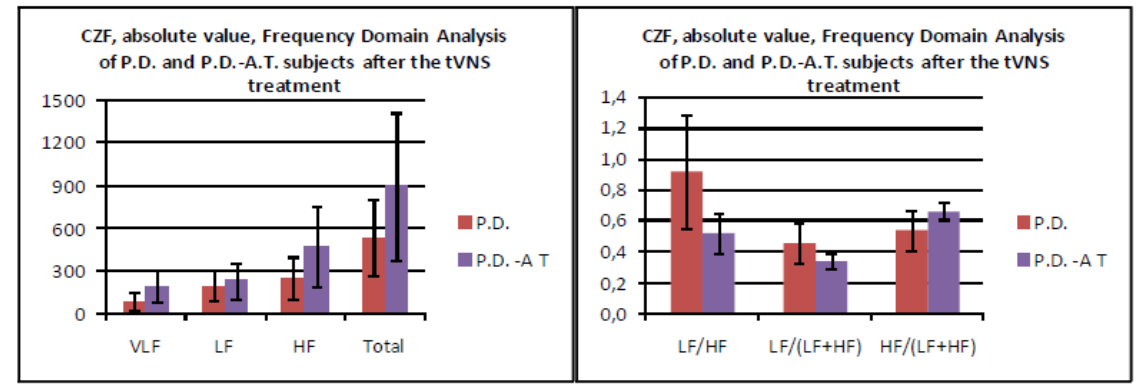

Figure 8: Let us examine the CZF method, both with absolute value and without absolute value.
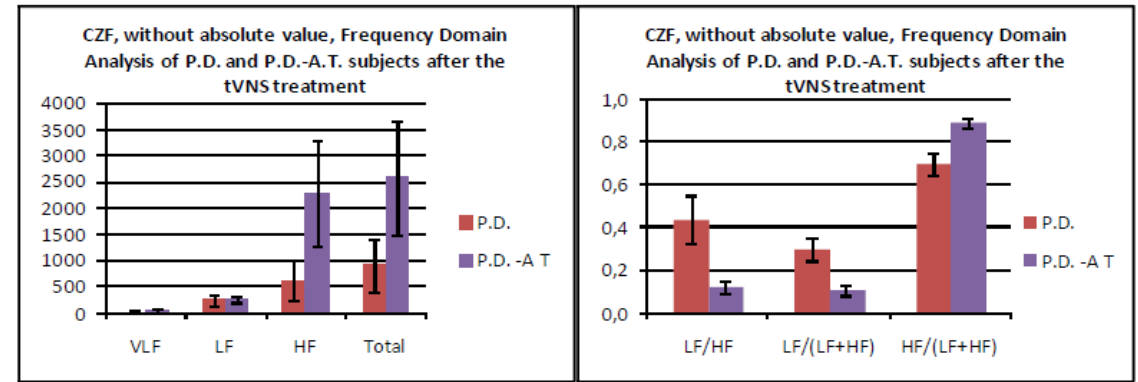

Figure 9: P.D. histograms are now pictured in brown for P.D. and purple for P.D.-AT

linked mainly to sympathetic modulation and baroreceptor activity, but also includes parasympathetic contributions. Finally, the HF spectral band reflects parasympathetic (vagal) activity.

b) We also used another method, the CZF method [13,15] to investigate variability of the R-R signal. We know that the standard FFT method in HRV contains limitations. The R-R time series is non stationary, non periodic and nonlinear. In principle, FFT should be applied only to linear, periodic and stationary signals. Consequently, basic information is missing, lost or approximated when using only FFT in HRV analysis. The situation improves by introducing some methodological innovation [13] based on appropriate physiological foundations. The combined use of two or more methods provides advancement in the reliability of the obtained results. A concept having valid support in physiology, in cardiology and in ANS and HRV studies, is the variability of heart rhythm. Variability may be formally expressed by $R R(n)-R R(n-k)$, where $R R(n)$ is the $R-R$ time interval value at the beat (n), and $R R(n-k)$ is the $R-R$ value at the beat $(\mathrm{n}-\mathrm{k})$. Considered values for $(\mathrm{k})$ are integers $(\mathrm{k}=1,2,3,4,5 \ldots)$ if we consider $\mathrm{k}=1$, we are estimating the difference (Variability) $(\mathrm{RR}(\mathrm{n})-\mathrm{RR}(\mathrm{n}-1))$ between two adjacent beats and for each beat $(\mathrm{n})$. The concept of variability is not new. In 1996, the Task Force [16] recommended using such a variable. To this regard, we also currently use the Poincare Plot in HRV analysis. However, it gives only a bidimensional nonlinear representation of variability by plotting the previous $\mathrm{R}-\mathrm{R}$ time value against the subsequent. It is unable to estimate VLF, LF and HF frequency domains. By using instead the CZF method, we estimate the previous conceptually-established idea of instantaneous variability of the RR(n) time series in the following manner:

$$
\operatorname{VaR}(n)=\frac{R R(n)(R R(n)-R R(n-1))}{\text { Mean value of } R R(n) \text { values }}
$$

The newly-obtained time series contains the difference (RR(n)$\mathrm{RR}(\mathrm{n}-1))$. It is the variability of R-R intervals, and may be estimated in absolute value (CZF-A.V.) and without absolute value (CZFW.A.V.). In the first case of (CZF-A.V.), we have the measure of 


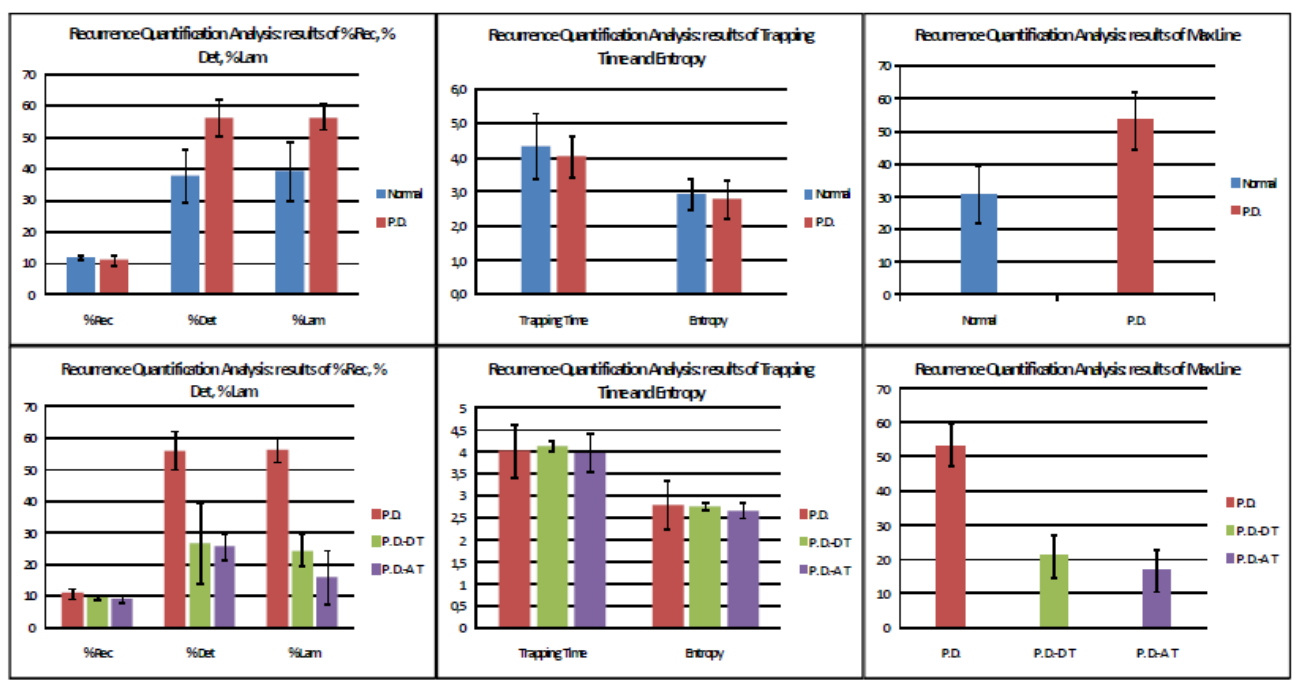

Figure 10: Compares the RQA results of normal subjects respect to P.D. subjects.

variability as the size of the variable itself. In the second case of (CZFW.A.V.), we estimate the dynamic heart rhythm profile, since we inspect when $(\mathrm{RR}(\mathrm{n})-\mathrm{RR}(\mathrm{n}-1))>0$ and when $(\mathrm{RR}(\mathrm{n})-\mathrm{RR}(\mathrm{n}-1))<0$. In this manner we have information on the dynamics between adjacent cardiac beats resulting, first, in a deceleration and, second, in an acceleration of the cardiac rhythm. Finally, performing the Discrete Fourier Transform (DFT) analysis on the time series (VaR(n)), we may evaluate variability in HRV in the three usual VLF, LF, and HF bands.

This method is of major importance in our study, since our aim is to analyze the variability induced in HR as a consequence of tVNS stimulation and, by this method, we have the possibility to compare it before, during, and after the treatment.

c) The final step of our investigation was realized by using the well-known method of Recurrence Quantification.

Analysis (R.Q.A.) [17]. This is a robust methodology that was originally was introduced by Zbilut and Webber was and subsequently elaborated by different authors [18-20], as we explain in detail in the next section. This method of non linear data analysis for investigation of dynamic systems has received, for many years, a constant and excellent international accreditation. It has been used by us in a number of papers [21].

\section{Results}

All the results related to the frequency domain analysis are given in Tables $1 \& 2$ for statistical significance. The values are given in $\mathrm{msec}^{2}$. Normal indicates states for normal subjects; P.D. indicates states for the subjects having psychological disorders. PD.DT relates the results obtained during the tVNS treatment and P.D.-A.T., those obtained soon after the treatment.

Let us start examining the results that we obtained before the tVNS treatment. In Figures 1 \& 2 we compare normal subjects against subjects with psychological disorders (P.D.) using the standard FFT and CZF methods.
By inspection of these results, we observe that all the P.D. subjects, as expected, showed increased values in the LF band, mainly related to increased sympathetic activity, and decreased values in the HF band (parasympathetic activity). The LF/HF, LF/(LF+HF), and $\mathrm{HF} /(\mathrm{LF}+\mathrm{HF})$ are strongly indicative of an increased activity of ANS modulation in the LF band, and decreased ANS modulation in the HF band, comparing P.D. subjects to normal subjects.

In Figure 2a, we pass to examining results of the variability of the R-R intervals by using the CZF method. We have the estimated values in absolute value. As previously explained, the use of the absolute value enables us to account for the size of the variability of adjacent beats, as given by the expression $(R R(n)-R R(n-1))$ for each beat $n$ ) (reflecting the behavior of the physiological dynamics.

By inspection of Figure 2, we deduce that in correspondence to an increased activity of ANS modulation in the P.D. subjects, as compared to the controls, we have a rather constant ANS balancing modulation of the heart rhythm, as expressed by the LF/HF, LF/(LF+HF), and $\mathrm{HF} /(\mathrm{LF}+\mathrm{HF})$ indexes. Therefore, a large compensation in size of variability is evidenced in ANS heart dynamics activity estimated in absolute value. Of course, this is not sufficient: intrinsic dynamics must be evaluated connecting to the previous analysis the real sign of the heart rhythm variability, considering the $(R R(n)-R R(n-1)$ differences with their real sign. This is to say, without absolute value. When positive, such a difference corresponds to an acceleration of the heartbeat. When such a difference is negative, it corresponds to a heartbeat deceleration induced by the ANS modulation.

Let us examine the results without absolute value as given in Figure 3 .

In this case, without absolute value, we evaluate the variability of the heart rhythm in its behavior. Compared to the controls, the P.D. subjects evidence a net increased variability in the LF band due to the increased sympathetic activity, while instead we verify a net decreased variability in the HF band due to parasympathetic activity. As consequence, the ratios LF/HF and LF/ (LF+HF) increase greatly in P.D. subjects, compared to the controls, while instead the ratio HF/ 
Table 1: Results of Frequency Domain Analysis in P.D, P.D.-DT, P.D.-AT Subjects in tVNS.

\begin{tabular}{|c|c|c|c|c|c|c|c|}
\hline & VLF & LF & $\mathrm{HF}$ & Total & $\mathrm{LF} / \mathrm{HF}$ & $\mathrm{LF} /(\mathrm{LF}+\mathrm{HF})$ & $\mathrm{HF} /(\mathrm{LF}+\mathrm{HF})$ \\
\hline \multicolumn{8}{|l|}{ F.F.T. } \\
\hline Normal & $536,50 \pm 347,46$ & $194,25 \pm 31,09$ & $187,50 \pm 48,25$ & $918,25 \pm 314,73$ & $1,07 \pm 0,41$ & $0,51 \pm 0,08$ & $0,48 \pm 0,08$ \\
\hline P. D. & $510,00 \pm 421,38$ & $582,66 \pm 572,342$ & $208,00 \pm 53,93$ & $1300,66 \pm 1179,67$ & $2,72 \pm 0,46$ & $0,72 \pm 0,03$ & $0,27 \pm 0,03$ \\
\hline P.D. -D T & $573,25 \pm 187,57$ & $340,75 \pm 76,19$ & $568,50 \pm 98,91$ & $1482,50 \pm 246,66$ & $0,57 \pm 0,05$ & $0,37 \pm 0,02$ & $0,62 \pm 0,02$ \\
\hline P.D. -A T & $775,75 \pm 480,26$ & $250,75 \pm 72,33$ & $671,50 \pm 383,63$ & $1698 \pm 767,46$ & $0,40 \pm 0,08$ & $0,28 \pm 0,06$ & $0,71 \pm 0,06$ \\
\hline \multicolumn{8}{|l|}{ CZF-A. V. } \\
\hline Normal & $75,15 \pm 60,73$ & $147,52 \pm 119,94$ & $177,62 \pm 172,56$ & $400,29 \pm 347,79$ & $0,922 \pm 0,19$ & $0,47 \pm 0,05$ & $0,52 \pm 0,05$ \\
\hline P. D. & $86,80 \pm 64,53$ & $198,56 \pm 104,00$ & $251,61 \pm 146,10$ & $536,99 \pm 271,67$ & $0,91 \pm 0,37$ & $0,46 \pm 0,12$ & $0,53 \pm 0,12$ \\
\hline P.D. -D T & $78,27 \pm 60,66$ & $126,97 \pm 62,59$ & $229,35 \pm 99,66$ & $434,60 \pm 206,48$ & $0,55 \pm 0,15$ & $0,35 \pm 0,06$ & $0,64 \pm 0,06$ \\
\hline P.D. -A T & $192,61 \pm 111,41$ & $232,966 \pm 127,25$ & $474,72 \pm 286,20$ & $900,31 \pm 521,77$ & $0,51 \pm 0,12$ & $0,33 \pm 0,05$ & $0,66 \pm 0,05$ \\
\hline \multicolumn{8}{|l|}{ CZF-W. A. V } \\
\hline Normal & $21,90 \pm 9,72$ & $155,00 \pm 75,20$ & $805,30 \pm 409,24$ & $982,21 \pm 788,79$ & $0,23 \pm 0,08$ & $0,18 \pm 0,05$ & $0,81 \pm 0,06$ \\
\hline P. D. & $25,61 \pm 10,60$ & $249,63 \pm 101,52$ & $631,67 \pm 391,33$ & $906,91 \pm 499,96$ & $0,43 \pm 0,11$ & $0,30 \pm 0,05$ & $0,69 \pm 0,05$ \\
\hline P.D. -D T & $19,06 \pm 6,50$ & $191,80 \pm 74,49$ & $1320,75 \pm 399,59$ & $1531,63 \pm 477,70$ & $0,14 \pm 0,01$ & $0,12 \pm 0,01$ & $0,87 \pm 0,01$ \\
\hline P.D. -A T & $41,47 \pm 28,02$ & $265,89 \pm 62,21$ & $2284,73 \pm 1003,18$ & $2592,11 \pm 1091,09$ & $0,12 \pm 0,02$ & $0,11 \pm 0,02$ & $0,88 \pm 0,02$ \\
\hline
\end{tabular}

Table 2: Statistical analysis: t-test.

\begin{tabular}{|c|c|c|c|c|c|c|c|c|}
\hline & Normal vs P. D. & P.D. vs P.D.-DT & P.D. vs P.D.-AT & P.D. - DT vs P.D.-AT & Normal vs P. D. & P.D. vs P.D.-DT & P.D. vs P.D.-AT & P.D. - DT vs P.D.-AT \\
\hline & FFT & & & & CZF - A. V. & & & \\
\hline LF & $p<0.001$ & $p<0.001$ & $p<0.001$ & $p<0.001$ & $p<0.05$ & $p<0.001$ & n.s. & $p<0.001$ \\
\hline $\mathrm{HF}$ & $p<0.05$ & $p<0.001$ & $p<0.001$ & $p<0.05$ & $p<0.05$ & n.s. & $p<0.001$ & $p<0.001$ \\
\hline Total & $p<0.001$ & n.s. & $p<0.05$ & $p<0.05$ & $p<0.05$ & $p<0.05$ & $p<0.001$ & $p<0.001$ \\
\hline \multirow[t]{9}{*}{ LF/HF } & $p<0.001$ & $p<0.001$ & $p<0.001$ & $p<0.001$ & n.s. & $p<0.001$ & $p<0.001$ & n.s. \\
\hline & $p<0.001$ & $p<0.001$ & $p<0.001$ & $p<0.001$ & n.s. & $p<0.001$ & $p<0.001$ & n.s. \\
\hline & $p<0.001$ & $p<0.001$ & $p<0.001$ & $p<0.001$ & n.s. & $p<0.001$ & $p<0.001$ & n.s. \\
\hline & CZF - W.A.V. & & & & & & & \\
\hline & LF & $p<0.001$ & $p<0.01$ & n.s & $p<0.001$ & & & \\
\hline & $\mathrm{HF}$ & $p<0.05$ & $p<0.001$ & $p<0.001$ & $p<0.001$ & & & \\
\hline & Total & n.s. & $p<0.001$ & $p<0.001$ & $\mathrm{p}<0.001$ & & & \\
\hline & $\mathrm{LF} /(\mathrm{LF}+\mathrm{HF})$ & $p<0.001$ & $p<0.001$ & $p<0.001$ & $p<0.001$ & & & \\
\hline & $\mathrm{HF} /(\mathrm{LF}+\mathrm{HF})$ & $p<0.001$ & $p<0.001$ & $p<0.001$ & $p<0.001$ & & & \\
\hline
\end{tabular}

(LF+HF) decreases.

In conclusion, in P.D. subjects, we have a dysfunction of the ANS modulation on heart rhythm, with increased activity of modulation in the LF band and decreased modulation in the HF band.

These arguments conclude our elaboration on our frequency analysis of control and P.D. subjects before starting the experiments with tVNS. We may now pass to examining the results during and after the vagus stimulation.

First, we have to say that in the controls (the normal subjects), the results during and after the tVNS did not show significant changes in the frequency domain. We concluded that giving a so weak current stimulation did not lead to significant changes in HRV in these subjects. Consequently, we will not consider them here. Instead, we obtained significant changes for P.D. subjects. The histograms are given, representing the results before and during the treatment in P.D. subjects and, soon after, before and after the treatment of these subjects. Results in the subjects before the treatment are represented by brown and during the treatment by green. Brown and purple are used when comparing results in the P.D. subjects before and after the tVNS treatment respectively.

First, let us study the results using the standard FFT. They are reported in Figure 4.

By inspecting Figure 4, we see that, in the VLF band, the results are moderately increased; in the LF band, we had a decrease of about $50 \%$; while in the HF band, we had an increase about $60 \%$. Consequently, the LF/HF ratio, starting with a mean value of 2.8 , resulted in a strong decrease to 0.6. The LF/ (LF+HF) ratio, starting with 0.7 in P.D., decreased to 0.35 ; and $\mathrm{HF} /(\mathrm{LF}+\mathrm{HF})$ increased to 0.65 . 
Table 3: Results of Quantification Recurrence Analysis in Normal, P.D., P.D.-D.T., P.D.-A.T. subjects

\begin{tabular}{|c|c|c|c|c|c|c|}
\hline & $\% \operatorname{Rec}$ & $\%$ Det & $\%$ Lam & Trapping Time & Entropy & Max Line \\
\hline Normal & $11,84 \pm 0,89$ & $37,78 \pm 8,33$ & $39,39 \pm 9,41$ & $4,33 \pm 0,96$ & $2,92 \pm 0,43$ & $31 \pm 8,87$ \\
\hline P.D. & $10,85 \pm 1,65$ & $56,26 \pm 5,89$ & $56,59 \pm 4,02$ & $4,03 \pm 0,59$ & $2,78 \pm 0,55$ & $54 \pm 7,87$ \\
\hline P. D.-D T & $9,45 \pm 0,75$ & $26,61 \pm 12,70$ & $24,54 \pm 4,89$ & $4,14 \pm 0,11$ & $2,77 \pm 0,08$ & $21 \pm 5,35$ \\
\hline P. D.-A T & $9,24 \pm 1,23$ & $25,77 \pm 4,00$ & $15,95 \pm 8,44$ & $3,97 \pm 0,42$ & $2,67 \pm 0,17$ & $17 \pm 6,2$ \\
\hline \multicolumn{7}{|c|}{ Statistical Analysis: t-test } \\
\hline \multicolumn{2}{|c|}{ Normal/P.D } & P.D./P.D.-DT & P.D.-DT/P.D.-AT & P.D./P.D.-AT & & \\
\hline \multicolumn{7}{|l|}{ RQA } \\
\hline$\% \operatorname{Rec}$ & $p<0.001$ & $p<0.001$ & n.s. & $p<0.001$ & & \\
\hline$\%$ Det & $p<0.001$ & $p<0.001$ & n.s. & $p<0.001$ & & \\
\hline$\%$ Lam & $p<0.001$ & $p<0.001$ & $p<0.001$ & $p<0.001$ & & \\
\hline Max Line & $p<0.001$ & $p<0.001$ & $p<0.001$ & $p<0.001$ & & \\
\hline
\end{tabular}

The conclusion is rather evident. The tVNS induced a profound decrease in sympathetic modulation and a corresponding net increase in parasympathetic modulation.

Let us examine now the results obtained by the CZF method, given in Figure 5, for the studied case with absolute value in the variability $(\mathrm{RR}(\mathrm{n})-\mathrm{RR}(\mathrm{n}-1)$

Inspection indicates a reduction in variability of about $40 \%$ in the LF band, compared to the P.D case before stimulation, and a moderate decrease of about $10 \%$ in the HF band. The ratios LF/HF and LF / (LF+HF) decreased, by $45 \%$ for the LF band and $25 \%$ for the $\mathrm{LF} /(\mathrm{LF}+\mathrm{HF})$ band, while there was a net increase of $30 \%$ for the HF band. In conclusion, in absolute value, the variability of heart rhythm increased using the tVNS, and the treatment substantially reduced the sympathetic component and enhanced the parasympathetic component.

In Figure 6, we give the results of the application to this case of the CZF method without absolute value thus accounting for acceleration and deceleration of the heart rhythm.

We see a reduction of more than $20 \%$ in the LF band and a strong enhancement, more than $50 \%$, in the HF band. The LF/HF ratio was reduced by about $60 \%$ and the $\mathrm{LF} /(\mathrm{LF}+\mathrm{HF})$ ratio by about $40 \%$, while instead the HF/ (LF+HF) ratio was increased by about $38 \%$. The results again confirm that during the tVNS treatment, we had a sustained activation of the parasympathetic component.

We have to examine now the results obtained after the tVNS treatment. Let us start with the standard FFT. The results are given in Figure 7.

These P.D.-AT (after treatment) results, completely confirm what we obtained during the treatment. We have an increased VLF, a strong reduction (over 60\%) in the LF band, and a corresponding increase in the $\mathrm{HF}$ band. There is also a strong reduction of the $\mathrm{LF} / \mathrm{HF}$ ratio and $\mathrm{LF} /(\mathrm{LF}+\mathrm{HF})$ ratios, and a strong increase in $\mathrm{HF} /(\mathrm{LF}+\mathrm{HF})$, respectively of $75 \%, 50 \%$, and $60 \%$.

Let us examine the CZF method, both with absolute value and without absolute value. The results are given in Figures 8 \& 9. Note that P.D. histograms are now pictured in brown for P.D. and purple for P.D.-AT.
There is only one conclusion. Apart from small fluctuations, there are no changes when comparing the results during and after the treatment. This confirms that the tVNS treatment manifests itself as a robust technique that maintains its effects after the treatment has been terminated.

We now have to examine results obtained by the Recurrence Quantification Analysis (RQA) method. As previously mentioned, RQA started with the study of the Recurrence Plot (RP) that was introduced in 1987 by Eckmann, Kamphorst, and Ruelle to represent a system behavior on the basis of recurrence theory, using phase space reconstruction of the given time series. In 1992, Zbilut and Webber developed a set of quantitative measures that are calculated in the terms of RP. They defined Recurrence Rate (RR), Determinism (DET), Divergence (DIV), entropy (ENTR) and trend (TREND). These measures formed the RQA method of analysis in the 1996 study by Trulla et al. In GAO, 1999 in 2002, Mar wan et al. worked out the measures of laminarity (LAM) and trapping time (TT).

1. RR represents the density of recurrence points in a recurrence plot (\%Rec.). It is the probability that a specific state will recur.

2. The $\%$ Det is the percentage of recurrence points which form diagonal lines in a recurrence plot of minimal length, and it is related to the predictability of the dynamic system.

3. \% LAM represents the amount of recurrence points which form vertical lines in the plot and is related to the amount of laminar phases in the system.

4. TT measures the average length of the vertical lines, and is related to the laminarity time of the dynamic system.

5. ENTR reflects the complexity of the deterministic structure in the system.

6. The last recurrence variable is the max line (LMAX), which is simply the length of the longest diagonal line segment in the plot, excluding the main diagonal line of identity $(i=j)$. This is a very important recurrence variable because it inversely scales with the most positive Lyapunov exponent [6-8,18,22-25].

The results of our experiment are reported in Table 3 for control 
subjects, and for P.D. subjects both during (P.D.-D.T.) and after the treatment (P.D.-A.T.). It is important to specify that, in order to perform the RQA, a phase space reconstruction of the tachograms is required. This enables us to fix a time delay, which we did by using the autocorrelation function and the Average Mutual Information. On this basis, the phase space reconstruction was realized by us using the criterion of the False Nearest Neighbors (F.N.N.). Our analysis enabled us to select a time delay of 2 , a dimension of 4 , a line of 3 , and a radius of 48 . For details, on the method see [17]. The results are given in Table 3.

Figure 10, top section, compares the RQA results of normal subjects respect to P.D. subjects. The analysis shows that in the P.D. case, we have a more marked \% Det and \% lam with a rather constant TT and, obviously, a reduced ENTR. The nonlinear system, regarding the P.D subjects, results more deterministic and predictable, with a more marked laminarity and a lightly reduced complexity. In addition, max line results increased, thus showing a markedly reduced complexity and chaoticity of P.D. compared to normal subjects.

In Figure 10, bottom section; we compare also the RQA results in P.D. subjects before, during, and after the treatment. We can see that, following the tVNS treatment, the \% Rec results are slightly reduced. The important feature is that \% Det and \% Lam results are profoundly reduced, maintaining both the trapping time and the entropy rather constant, but with the tendency of the system to recover in complexity, as confirmed by max line results. In brief, the tVNS enables the P.D. subjects to recover and gain in complexity and variability -chaoticity.

\section{Conclusion}

Transcutaneous Vagus Nerve Stimulation (tVNS) results to be a treatment that operates delivering electrical impulses to the vagus nerve, and currently may be applied in a very large body of medical and, in particular, physiological and psycho-physiological fields. Results are needed that quantify in detail the intrinsic dynamics from an electrophysiological point of view, and examine the validity of the devices that are currently employed. A number of methodological studies is required in order to understand and quantify in detail as it operates in the single treatment. Without doubt the methodological analysis of HRV during the t-VNS single treatment represents the first requirement in this approach. This is the reason because we performed the HRV -frequency domain analysis during the t-VNS treatment using the standard FFT methods and, as said, improving the analysis of the data by using the method of analysis of variability (CZF method). To investigate the non linear presence we used the Recurrence Quantification Analysis (RQA). Our methodological conclusion is that the tVNS does not affect normal subjects in relation to HRV. However, in subjects that started with a serious dysfunction of the ANS, as mirrored by the HRV, and evidencing a net dominance of the LF band (as observed in HRV), the results of their tVNS treatment showed a net improvement of the ANS in the HRV, with an evident recovered ANS balance and/or dominance of the HF band. This is the technical result of our experimentation corresponding of course to the aim of our work. Since we applied only one t-VNS treatment we did not expect to observe immediate and net improvements also under the general psychological conditions of subjects and therefore we did not deepen these features by performing new psychological tests. The important feature that our staff characterized in detail is that the subjects did not suffer negative side effects to any degree after the treatment and all the tested patients reported a subjective amelioration, and in particular of their mood, after the treatment.

\section{References}

1. Fink M. The origins of convulsive therapy. Am J Psychiatry. 1984; 141: 10341041.

2. Schachter SC, Saper CB. Vagus nerve stimulation. Epilepsia. 1998; 39: 677686.

3. Ben-Menachem E. Vagus- nerve stimulation for the treatment of epilepsy. Lancet Neurol. 2002; 1: 477-482.

4. Grimm S, Bajbouj M. Efficacy of vagus nerve stimulation in the treatment of depression. Expert Rev. Neurother. 2010; 10: 87-92.

5. Schwartz PJ, De Ferrari GM, Sanzo A, Landolina M, Rordorf R, Raineri C, et al. Long-term vagal stimulation in patients with advanced heart failure: first experience in man. Eur J Heart Fail. 2008; 10: 884-891.

6. De Ferrari GM, Crijns HJ, Borggrefe M, Milasinovic G, Smid J, Zabel M, et al. Chronic vagus nerve stimulation: a new and promising therapeutic approach for chronic heart failure. Eur Heart J. 2011; 32: 847-855.

7. Chae JH, Nahas Z, Lomarev M, Denslow S, Lorberbaum JP, Bohning DE, et al. A review of functional neuroimaging studies of vagus nerve stimulation (VNS). J Psychiatr Res. 2003; 37: 443-455.

8. Pardo JV, Sheikh SA, Schwindt GC, Lee JT, Kuskowski MA, Surerus C, et al. Chronic vagus nerve stimulation for treatmentresistant depression decreases resting ventromedial prefrontal glucose metabolism. Neuroimage. 2008; 42 : 879-889.

9. Vonck K, De Herdt V, Bosman T, Dedeurwaerdere S, Van Laere K, Boon P Thalamic and limbic involvement in the mechanism of action of vagus nerve stimulation, a SPECT study. Seizure. 2008; 17: 699-706.

10. Kosel M, Brockmann H, Frick C, Zobel A, Schlaepfer TE. Chronic vagus nerve stimulation for treatmentresistant depression increases regional cerebral blood flow in the dorsolateral prefrontal cortex. Psychiatry Res. 2011; 191 . 153-159.

11. Ell rich J. Transcutaneous vagus nerve stimulation Eur. Neurol. Rev. 2011; 6: $262-264$

12. Kraus T, Hosl K, Kiess O, Schanze A, Kornhuber J, Forster C. BOLD f MR deactivation of limbic and temporal brain structures and mood enhancing effect by transcutaneous vagus nerve stimulation. J Neural Transm. 2007; 114: $1485-1493$

13. Conte E, Giroldini W, Laterza V, Conte S, Pieralice M, Casciaro F, et al. Experimental Results on a New Method for Analysis of Heart Rate Variability. World Journal of Cardiovascular Diseases. 2014; 4: 385-389.

14. Conte E, Federici A, Ziblut JP. A new method based on fractal variance function for analysis and quantification of sympathetic and vagal activity in variability of R-R time series in ECG signals. Chaos Solitons \& Fractals. 2009; 41: $1416-1426$

15. Conte E, Khrenniokov A, Federici A, Zbilut JP. Fractal fluctuations and quantum-like chaos in the brain by analysis of variability of brain waves: $A$ new method based on a fractal variance function and random matrix theory: $A$ link with El Naschie fractal Cantorian space-time and V. Weiss and $\mathrm{H}$. Weiss golden ratio in brain. Chaos Solitons \& Fractals. 2009; 41: 2790-2800.

16. Task Force of the European Society of Cardiology and the North American Society of Pacing and Electrophysiology Heart Rate Variability: Standards of Measurement, Physiological Interpretation and Clinical Use. Circulation. 1996; 93: 1043-1065.

17. Zbilut JP, Webber CL. Embedding and delays as derived from quantification of Recurrence Plot. Phys Lett A. 1992; 171: 199-203.

18. Trulla LL, Giuliani A, Zbilut JP, Webber CL. Recurrence quantification analysis of the logistic equation with transients. Phys Lett A. 1996; 223: 255-260.

19. Gao JB. Recurrence time statistics for chaotic systems and their applications. Phys Rev Let. 1999; 83: 3178-3181. 
20. Marwan N, Romano MC, Thiel M, Kurths J. Recurrence plots for the analysis of complex systems. Physics Reports. 2007; 438: 237-329.

21. Conte E, Federici A, Minervini M, Papagni A, Zbilut JP. Measures of coupling strength and synchronization in nonlinear interaction of heart rate and systolic blood pressure in the cardiovascular control system. Chaos and Complexity Letters. 2008; 2: 1-22.

22. Eckmann JP, Kamphorst SO, Ruelle D. Recurrence Plot of dynamical system. Europhys Let. 1987; 4: 973-977.
23. Vonck K, Boon $\mathrm{P}$, Van Roost $\mathrm{D}$. Anatomical and physiological basis and mechanism of action of neuron stimulation for epilepsy. Acta Neurochir Suppl. 2007; 97: 321-328.

24. Peng CK, Buldyrev SV, Havlin S, Simons M, Stanley HE, Goldberger AL. Mosaic organization of DNA. Sequences Phys Rev E. 1994; 49: 1685-1689.

25. Hurst HE. Long-term storage capacity in reservoirs. Transactions of American Society of Civil Engineers. 1951; 55: 400- 410.
Ann Depress Anxiety - Volume 5 Issue 1 - 2018

ISSN : 2381-8883 | www.austinpublishing group.com

Conte et al. (C) All rights are reserved
Citation: Conte S, Wang F, Altamura M, Bellomo A, Serafini G, Orsucci F, et al. Analysis of Heart Rate variability (HRV) in Frequency Domain and Recurrence Quantification Analysis during the Treatment by Transcutaneous Vagus Nerve Stimulation. Ann Depress Anxiety. 2018; 5(1): 1093. 